Particle Accelerators, 1987, Vol. 22, pp. 15-34

Photocopying permitted by license only

(C) 1987 Gordon and Breach Science Publishers, Inc.

Printed in the United States of America

\title{
LIE ALGEBRAIC MAPS AND INVARIANTS PRODUCED BY TRACKING CODES
}

\author{
ETIENNE FOREST \\ SSC Central Design Group, c/o Lawrence Berkeley Laboratory, \\ Berkeley, CA 94720
}

(Received July 24, 1986; in final form December 1, 1986)

We have derived the tools necessary for the extraction of a map from standard tracking programs using multipole kicks. Our results apply to programs using approximate Hamiltonians (PATRICIA, RACETRACK) or to the more rigorous symplectic integrators. As an example of a symplectic integrator, we chose the kick code TEAPOT for its simplicity. Our results are nevertheless general, because Ruth and Neri have shown that symplectic integrators are, in fact, kick codes. Finally, we give the general formulas for geometric invariants which are quartic extensions of the usual Courant-Snyder invariants. It should be noted that invariants are usually computed from the Hamiltonian. In accelerator physics, that requires a path length-dependent perturbation theory. Instead, the use of a mapping eliminates the path length dependence. Hence, the search for nonlinear invariants reduces to a problem similar to the diagonalization of a linear matrix.

\section{INTRODUCTION}

In the study of circular machines, fast methods of tracking have always been useful. For large, error-dominated rings, such as the contemplated SSC, one must take into account the effect of very high-order multipoles.

Traditionally, the multipoles have been incorporated into tracking programs as zero-length elements or kicks. Unfortunately, these kick codes have tracking as their primary goal and are incapable of giving certain insights into the nature of the nonlinear lattice functions. On the other hand, matrix codes such as MARYLIE $^{1}$ or TRANSPORT $^{2,3}$ have the capability of computing nonlinear lattice functions ${ }^{4}$ around the design orbit. Unfortunately, they have no way of computing exactly the matrices around an arbitrary orbit. This is because of the perturbative aproach of these codes; they expand everything around the design trajectory. Off-momentum effects, as well as misalignments, are not treated exactly.

In this paper, we intend to show how one computes the nonlinear map around any trajectory with the help of a kick code. From the nonlinear map, represented by Lie transforms, we will extract the nonlinear invariants. The maps computed by our method from kick codes are accurate to third order around the chosen trajectory, but, unlike those of matrix codes, they are exact in the momentum and the magnitude of the misalignments of the orbit under study. For a standard treatment using the Hamiltonian, the reader should consult Refs 5 and 6. 


\subsection{The Choice of a Coordinate System}

The orbit we choose to study will be represented by a vector $Z$ :

$$
Z=\left(X, P_{x}, Y, P_{y}, T, P_{t}\right) .
$$

The positions $X$ and $Y$ are deviations from the ideal closed orbit of the machine. Often they are chosen in a plane perpendicular to the design momentum, but as we will discover, this is not always necessary. The momentum $P_{x}$ and $P_{y}$ are also in the plane of $(X, Y)$. They measure the deviation from the ideal momentum $P_{0}$ and they are scaled by $P_{0}$. The time $T$ is defined as

$$
T=c \times \text { time of flight, }
$$

where $c=$ speed of light. Finally, $P_{t}$ is related to the energy deviation:

$$
P_{t}=-\left(\frac{E-E_{0}}{c P_{0}}\right)
$$

\subsection{Is $\left(P_{x}, P_{y}\right)$ Better than $\left(X^{\prime}, Y^{\prime}\right)$ ?}

Most kick codes use $X^{\prime}(=d x / d s)$ and $Y^{\prime}$ instead of the momenta $P_{x}$ and $P_{y}$. The use of $X^{\prime}$ and $Y^{\prime}$ facilitates the expression of the map in a drift space:

$$
\begin{aligned}
& \Delta X=L X^{\prime}, \\
& \Delta Y=L Y^{\prime},
\end{aligned}
$$

where $L=$ path length of the design orbit. Since Eq. (4) is exact, it implies that, in terms of the momenta, drifts are more complex. In fact, one needs to replace $X^{\prime}$ and $Y^{\prime}$ by the expressions

$$
\begin{aligned}
X^{\prime} & =\frac{P_{x}}{P_{s}}, \\
Y^{\prime} & =\frac{P_{y}}{P_{s}}, \\
P_{s} & =\left(1-\frac{2 P_{t}}{\beta_{0}}+P_{t}^{2}-P_{x}^{2}-P_{y}^{2}\right)^{1 / 2} .
\end{aligned}
$$

On the other hand, multipole kicks are much simpler in terms of the momentum:

$$
\begin{gathered}
\Delta \mathbf{P}=\frac{q L}{P_{0}}\left[A_{s}, \mathbf{P}\right], \\
\delta(s) A_{s}(\mathbf{r})=\text { vector potential, } \\
\mathbf{r}=(X, Y) \text { measured in the plane of the multipole. }
\end{gathered}
$$

As the reader can see, one cannot win. For $\left(X^{\prime}, Y^{\prime}\right)$ the drifts are trivial, while for a canonical set, the kicks are straightforward. 


\section{Making $X^{\prime}$ and $Y^{\prime}$ Canonical by Approximating Kicks}

the case of a large machine, where the influence of a single kick is small, it is nventional to introduce an approximation:

$$
\begin{aligned}
X^{\prime} & =\frac{P_{x}}{P_{s}} \simeq \frac{P_{x}}{\left(1-\frac{2 P_{t}}{\beta_{0}}+P_{t}^{2}\right)^{1 / 2}}=\frac{P_{x}}{(1+\delta)}, \\
\delta & =\frac{P-P_{0}}{P_{0}} .
\end{aligned}
$$

th this approximation, the equation for a kick becomes

$$
\Delta \mathbf{r}^{\prime}=\frac{q L}{P_{0}(1+\delta)}\left[A_{s}, \mathbf{P}\right] .
$$

nsistent with this approximation, one writes down a Hamiltonian in terms of For example, in a straight element, the correct Hamiltonian $H$ is given by

$$
H=P_{s}-\frac{q}{P_{0}} A_{s} .
$$

t, following Eq. (7) we approximate Eq. (9) as follows:

$$
\begin{gathered}
H=-(1+\delta)\left(1-\frac{P_{x}^{2}+P_{y}^{2}}{(1+\delta)^{2}}\right)^{1 / 2}-\frac{q}{P_{0}} A_{s}, \\
\tilde{H} \simeq-(1+\delta)+\frac{1}{2(1+\delta)}\left(P_{x}^{2}+P_{y}^{2}\right)-\frac{q}{P_{0}} A_{s} .
\end{gathered}
$$

if one does not need the time of flight information contained in the $\delta$ sendence of $H$ and $\tilde{H}$, one can replace $\mathbf{P}$ by $\mathbf{r}^{\prime}$. The Hamiltonian $\tilde{K}$ governing motion of $\left(\mathbf{r}, \mathbf{r}^{\prime}\right)$ is just

$$
\tilde{K}=\frac{\tilde{H}+(1+\delta)}{(1+\delta)}=\frac{1}{2}\left(X^{\prime 2}+Y^{\prime 2}\right)-\frac{q}{P_{0}(1+\delta)} A_{s} .
$$

e Hamiltonian $\tilde{K}$ is at the root of many traditional codes used in accelerator iign. These include PATRICIA ${ }^{7}$ and RACETRACK ${ }^{8}$. The nonlinear tools ich are discussed in the later sections of this paper have been implemented in program RACETRACK to fourth order. We are also working in collaboraa with Alex Dragt and Filippo Neri to extend these methods to fifth order in program RACETRACK.

n $\tilde{K}$ the quantity $\delta$ is just a parameter. For a full six-dimensional treatment, : must rely on $\tilde{H}$. For this reason, we have rescaled the matrices in ،CETRACK to correspond to $\tilde{H}$. The Hamiltonian $\tilde{K}$ (as well as $\tilde{H}$ ) introduce ors which are proportional to the integrated strength of $A_{s}$. It provides a very urate chromatic description of very large machines because the use of $X^{\prime}$ and does not introduce errors solely proportional to the length of the ring. On the 
other hand, codes using the correct expressions for $P_{x}$ and $P_{y}$ introduce errors which are of zeroth order in the length any time they approximate the drift [Eq. (5)] by a Taylor series (MARYLIE for example).

Of course, for a small ring, the integrated strength of $A_{s}$ can be large. Dragt noticed in the context of the Los Alamos Proton Storage Ring (PSR) the inadequacy of $\tilde{K}$ in predicting the correct chromatic properties. ${ }^{9}$ This is described in Ref. 10.

\subsection{The No-Compromise Kick Code: TEAPOT}

Passing from $\left(X^{\prime}, Y^{\prime}\right)$ to $\left(P_{x}, P_{y}\right)$ involves a square root only. If one is willing to live with it, it is possible to write a kick code which uses the exact formulas for the drift and the multipole kicks. Such a code has no dynamical approximation of the type introduced in Section 1.3. Its only approximation is to replace an ideal long element by one or a few thin-lens kicks represented by Eq. (6).

It is important to emphasize that it would be possible to regain long elements by splitting them into a large number of kicks and drifts. This is not the case if an incorrect Hamiltonian such as $\tilde{K}$ is used to describe the thin lenses. In fact, if one uses $\tilde{K}$ for a long element, only the linear part of the map is correctly restored. (See Ref. 10.)

Inspired by the desire to be exact, Schachinger and Talman, have written a code which uses the exact formalism for the lenses. This program, TEAPOT ${ }^{10}$ (Thin Element Accelerator Program for Optics and Tracking), has been equipped with an algorithm (LIEPOT) capable of generating a six-dimensional map around any orbit. As a result of the strict compliance of TEAPOT to the thin-lens model, we have been able to use the concatenation routines of the program MARYLIE in the map-generating algorithm.

In the next section, we examine the tools necessary to extract a map from a kick code. We concentrate on the example of TEAPOT, because of its greater complexity and exactness.

\section{THE ELEMENTS OF A THIN-LENS CODE}

The present version of TEAPOT contains multipole kicks, drifts, -and rotations in the plane of the ring. It has also a special linear kick to handle sector bends. The basic ideas of the code are contained in Fig. 1. This figure shows two successive multipole planes $\mathscr{P}_{i}$ and $\mathscr{P}_{i+1}$. The center of the multipoles are located at $P_{i}$ and $P_{i+1}$. The canonical coordinates of the particles at plane $\mathscr{P}_{i}$ are expressed with respect to $P_{i}$ in a frame within $\mathscr{P}_{i}$. The $x_{i}$ direction points away from the center of the multipole, and $y_{i}$ is perpendicular to the plane of the ring. The components of $P_{i+1}$ in the frame of $\mathscr{P}_{i}$ are given by the pair $\left(\mathscr{Y}_{i+}, \mathscr{X}_{i+}\right)$.

The particle drifts towards $\mathscr{P}_{i+1}$. Finally, $X_{i+1}$ and $Y_{i+1}$ are the coordinates of the particle expressed in the plane $\mathscr{P}_{i+1}$ just before entering the next multipole. Notice that we use capital letters for the ray under study, unlike Ref. 10, where lowercase letters are used. 


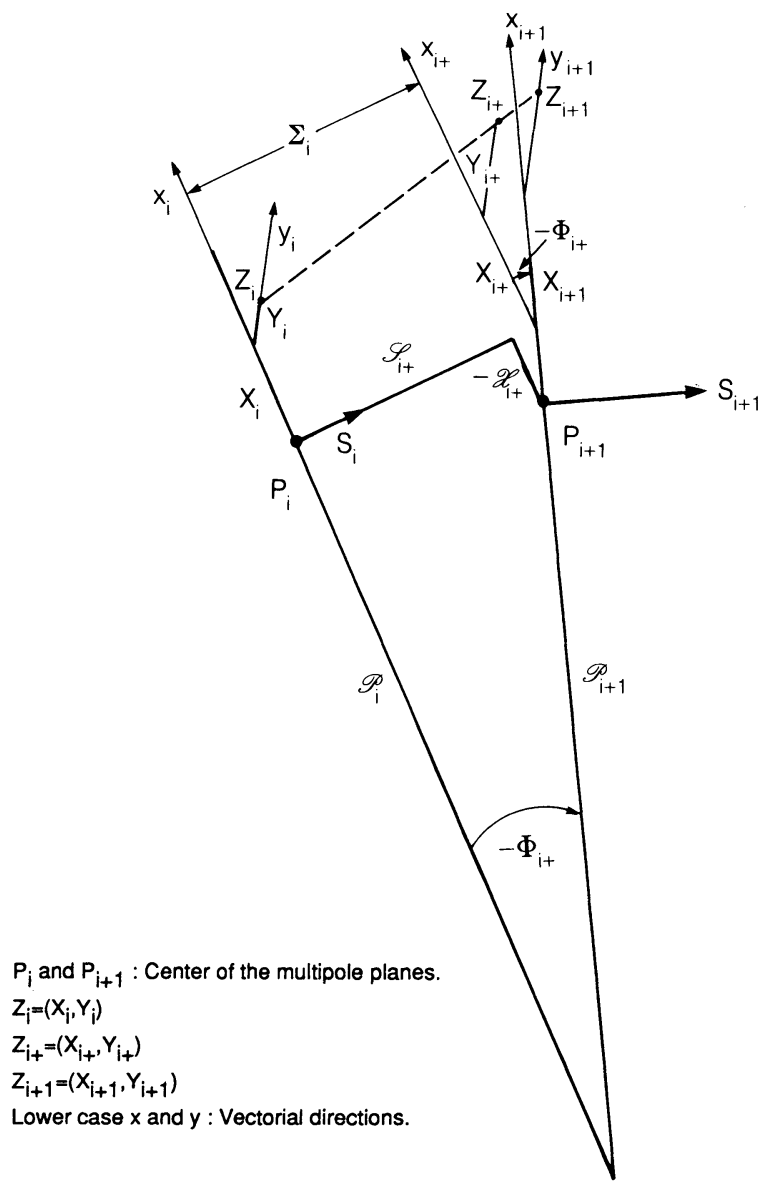

FIGURE 1 The geometry for straight line propagation through a drift space in the program TEAPOT.

\subsection{The Tracking in TEAPOT}

Our description of the tracking procedure differs from that of Ref. 10 by its use of canonical variables. Furthermore, here the two steps involved are symplectic (i.e., derivable from a Hamiltonian). In TEAPOT the steps used in the tracking, when analyzed individually, cannot be described by a Hamiltonian. Ultimately, however, the two descriptions are completely equivalent.

First, it is instructive to derive the connection between $\left(X_{i}, Y_{i}, T_{i}\right)$ and $\left(X_{i+1}, Y_{i+1}, T_{i+1}\right)$ in terms of canonical transformations. Consider the phase-space vector $Z_{i}$ defined in Section 1:

$$
Z_{i}=\left(X_{i}, P_{x i}, Y_{i}, P_{y i}, T_{i}, P_{t i}\right)
$$


The particle drifts in the frame of $\mathscr{P}_{i}$ a distance $\Sigma_{\mathrm{i}}$ :

$$
\begin{gathered}
\Sigma_{i}=\mathscr{Y}_{i+}+\mathscr{X}_{i+} \tan \phi_{i+}, \\
X_{i+}=X_{i}+\frac{P_{x i}}{P_{s i}} \Sigma_{i}, \\
Y_{i+}=Y_{i}+\frac{P_{y i}}{P_{s i}} \Sigma_{i}, \\
T_{i+}=T_{i}+\left(\frac{\frac{1}{\beta_{0}}-P_{t i}}{P_{s i}}\right) \Sigma_{i}, \\
P_{s i}=\left(1-\frac{2}{\beta_{0}} P_{t i}+P_{t i}^{2}-P_{x i}^{2}-P_{y i}^{2}\right)^{1 / 2} .
\end{gathered}
$$

All the momenta are left invariant by the drift. We then rotate $Z_{i+}$ in the frame of $\mathscr{P}_{i+1}$ and express the component $X_{i+1}$ with respect to the point $P_{i+1}$ :

$$
\begin{gathered}
X_{i+1}=\frac{X_{i+}}{\left(1+\frac{P_{x i}}{P_{s i}} \tan \phi_{i+}\right) \cos \phi_{i+}}-\frac{\mathscr{X}_{i+}}{\cos \phi_{i+}}, \\
P_{x(i+1)}=\cos \phi_{i+} P_{x i}-\sin \phi_{i+} P_{s i}, \\
Y_{i+1}=Y_{i+}-\frac{\tan \phi_{i+} X_{i+}}{1+\frac{P_{x i}}{P_{s i}} \tan \phi_{i+}} \frac{P_{y i}}{P_{s i}}, \\
P_{y(i+1)}=P_{y i}, \\
T_{i+1}=T_{i+}+\frac{\tan \phi_{i+}}{1+\frac{P_{x i}}{P_{s i}} \tan \phi_{i+}} \frac{\left(\frac{1}{\beta_{0}}-P_{t i}\right)}{P_{s i}} X_{i+} .
\end{gathered}
$$

Substitution of Eqs. (13) into Eqs. (14) leads to the expressions used in the program TEAPOT:

$$
\begin{gathered}
X_{i+1}=\frac{1}{\cos \phi_{i+}} \frac{X_{i}-\mathscr{X}_{i+}+\frac{P_{x i}}{P_{s i}} \mathscr{Y}_{i+}}{1+\frac{P_{x i}}{P_{s i}} \tan \phi_{i+}}, \\
P_{x(i+1)}=\cos \phi_{i+} P_{x i}-\sin \phi_{i+} P_{s i}, \\
Y_{i+1}=Y_{i+}+\frac{P_{y i}}{P_{s i}} \frac{\mathscr{S}_{i+}-\tan \phi_{i+}\left(X_{i}-\mathscr{X}_{i+}\right)}{1+\frac{P_{x i}}{P_{s i}} \tan \phi_{i+}}, \\
P_{y(i+1)}=P_{y i},
\end{gathered}
$$




$$
\begin{gathered}
T_{i+1}=T_{i}+\left(\frac{\frac{1}{\beta_{0}}-P_{t i}}{P_{s i}}\right) \Sigma_{i}+\frac{\tan \phi_{i+}}{1+\frac{P_{x i}}{P_{s i}} \tan \phi_{i+}} \frac{\left(\frac{1}{\beta_{0}}-P_{t i}\right)}{P_{s i}}\left(X_{i}+\frac{P_{x i}}{P_{s i}} \Sigma_{i}\right) \\
P_{t(i+1)}=P_{t i} .
\end{gathered}
$$

Finally, we consider the multipole kick. In terms "of the canonical vector $Z$, kicks are represented by the potential $\tilde{A}$ :

$$
\begin{gathered}
\tilde{A}=\frac{-q L B}{P_{0}} \operatorname{Re} \sum_{n=0}^{\infty} \frac{1}{(n+1)}\left(i a_{n}+b_{n}\right)(X+i Y)^{n+1}, \\
Z^{+}=\exp \left(: \tilde{A}\left(Z^{-}\right):\right) Z^{-}=Z^{-}+\left[\tilde{A}, Z^{-}\right] .
\end{gathered}
$$

The program allows for misalignments in the plane of the multipole. Rotation errors are obtained by modifying $a_{n}$ and $b_{n}$. Translation errors are computed by translating $\tilde{A}$ :

$$
\tilde{A}_{\text {errors }}=\tilde{A}\left(X^{-}-\Delta X^{-}, Y^{-}-\Delta Y^{-}\right) .
$$

It should be added that sector bends are treated by including an extra term in $\tilde{A}$.

$$
\tilde{A}_{\text {errors }}=A+\alpha\left(X^{-}-\Delta X^{-}\right)+\beta\left(Y^{-}-\Delta Y^{-}\right) \text {. }
$$

This completes the description of this kick code in terms of canonical variables. We now look at the expansion around a trajectory.

\subsection{The Transfer Map Expanded around a Trajectory: LIEPOT}

The expansion of a map around a given trajectory involves taking the derivatives of the tracking operations of a given program. One follows a particle along its path and simultaneously differentiates the operations performed by the code. The resulting matrix is concatenated with the matrices of the previous operations. At the end, one gets a first-, second-, or even third-order matrix for the full ring relative to the given trajectory. This brute force method has been incorporated in the programs MATPOT (matrix TEAPOT) and MATRACE(Matrix Racetrack) to third order in $\left(X, P_{x}, Y, P_{y}\right)$. It should be noted that a Lie algebraic representation can be extracted from the matrix representation of the map. ${ }^{11}$

Here we will be more elegant and perhaps more confusing. Instead of a direct differentiation of the expressions in Section 2.1, we appeal to elementary geometry in defining the nonlinear maps around a given trajectory. The map representing the motion of a particle from one plane to the next is a product of a rotation, a drift, and a second rotation. The parameters defining this map will depend on the particular ray for which the map is computed. Finally, our treatment is six-dimensional and Lie algebraic from the start. With this in mind, the reader is invited to carefully follow the description of these three operations with Fig. 2 as a guide. 


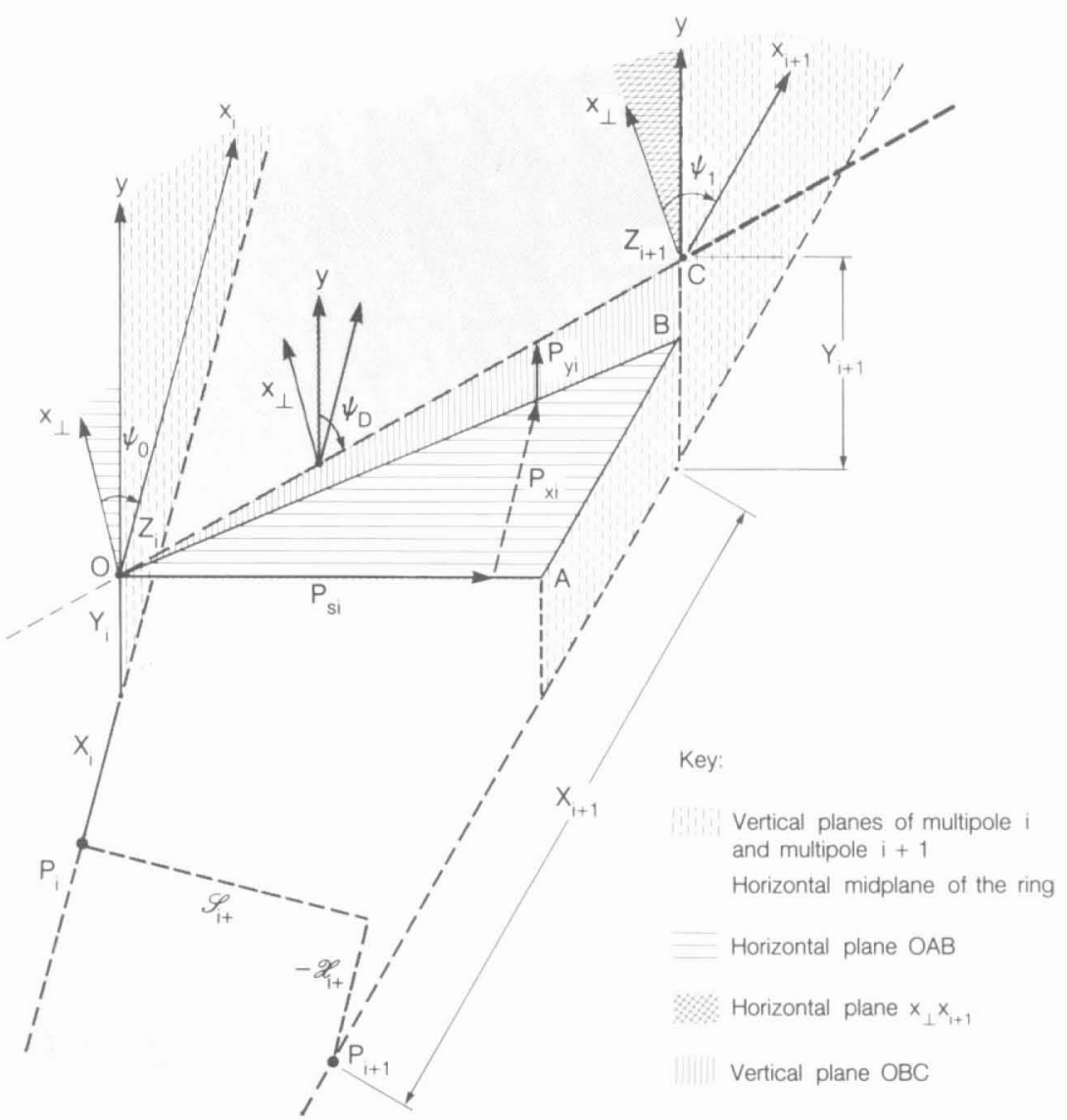

FIGURE 2 The geometry involved in the computation of the nonlinear map around an arbitrary trajectory in the program LIEPOT.

A. The drift between the multipole planes. Consider an arbitrary trajectory $Z_{i}$ at the plane $\mathscr{P}_{i}$, as shown on Fig. 2:

$$
Z_{i}=\left(X_{i}, P_{x i}, Y_{i}, P_{y i}, T_{i}, P_{t i}\right) \text {. }
$$

Suppose we are interested in a neighboring ray $Z$ :

$$
Z=Z_{i}+\zeta_{i} ; \zeta_{i}=\left(x_{i}, p_{x i}, y_{i}, p_{y i}, \tau_{i}, p_{\tau i}\right)
$$

For simplicity, we will scale $\zeta_{i}$ by $P_{0 i}$, where $P_{0 i}$ is the momentum of $Z_{i}\left(Z_{i}\right.$ is scaled by $P_{0}$, the momentum of the design trajectory of the machine). The variable $\zeta_{i}$ is measured in the plane $\mathscr{P}_{i}$.

Now, consider a rotation of angle $\psi_{0}$ in the plane of the ring. We choose $\psi_{0}$ such that the new frame has its $x$ axis perpendicular to the projection of the ray in the plane of the ring:

$$
\tan \psi_{0}=\frac{P_{x i}}{P_{s i}}
$$

Such a rotation will depend on $\psi_{0}, P_{y i}$, and $V_{0 i} / c$, the velocity of the ray $Z_{i}$. Let 
us assume that it can be represented by a symplectic operator $\mathscr{R}\left(\psi_{0}, P_{y i}\right)$ :

$$
\zeta_{\perp}=\mathscr{R}^{-1}\left(\psi_{0}, P_{y i}\right) \zeta_{i} \text {. }
$$

By writing the inverse of $\mathscr{R}$ in Eq. (21), we assume that $\mathscr{R}$ rotates from a perpendicular frame to an arbitrary frame. The Lie representation ${ }^{12}$ for $\mathscr{R}$ has the form

$$
\mathscr{R}=\mathscr{F}_{2} e^{: f_{3}}: e^{: f_{4}: \mathscr{G}_{2}}
$$

The expressions for $\mathscr{F}_{2}, \mathscr{G}_{2}, f_{3}$, and $f_{4}$ are given in Appendix A, Table A-II.

As shown in Fig. $2, \zeta_{\perp}$ is such that its $y$ axis makes an angle $\psi_{D}$ with the ray $Z_{i}$ :

$$
\psi_{D}=\tan ^{-1} \frac{P_{y i}}{\left(P_{s i}^{2}+P_{x i}^{2}\right)^{1 / 2}} \text {. }
$$

We can derive a symplectic transformation which corresponds to the drift around $Z_{i}$. In the frame of $\zeta_{\perp}$, it is given by the map $\mathscr{D}$ :

$$
\zeta_{\perp}^{\text {final }}=\mathscr{D}\left(O B, P_{y i}\right) \zeta_{\perp} \text {. }
$$

As hinted in Eq. (24), $\mathscr{D}$ will depend on the distance $O B$ in the plane $O A B$, as well as the vertical momentum of the ray $Z_{i}$. The Lie algebraic representation of $\mathscr{D}$ is given in Appendix A, Table A-I.

Finally, we can use $\mathscr{R}$ to rotate $\zeta_{\perp}$ into the plane $\mathscr{P}_{i+1}$. This involves a rotation by an angle $\psi_{1}$ :

$$
\psi_{1}=\psi_{0}-\phi_{i+} .
$$

We can express the full map from $\zeta_{i}$ to $\zeta_{i+1}$ as follows:

$$
\zeta_{i+1}=\mathscr{R}^{-1}\left(\psi_{0}, P_{y i}\right) \mathscr{D}\left(O B, P_{y i}\right) \mathscr{R}\left(\psi_{1}, P_{y i}\right) \zeta_{i} \text {. }
$$

$B$. The multipole kick. The Lie algebraic representation of a multipole kick in terms of $\zeta_{i}$ is obtainable from Eq. (17). In fact, we need only to expand $\tilde{A}_{\text {errors }}$ around $Z_{i}$ in homogeneous polynomials in $\zeta_{i}$ :

$$
\begin{gathered}
f_{m}\left(\zeta_{i}\right)=\tilde{A}_{\text {errors }}^{m}\left(Z_{i} ; \zeta_{i}\right) \quad(m \geq 2), \\
\tilde{A}_{\text {errors }}\left(Z_{i}+\zeta_{i}\right)=\sum_{m=0}^{\infty} \tilde{A}^{m}\left(Z_{i} ; \zeta_{i}\right) .
\end{gathered}
$$

The computation of each $f_{m}$ requires the evaluation of only two derivatives. This is a result of Maxwell's equations. In fact, the computation of these two derivatives is equivalent to computing $B_{x}$ and $B_{y}$ with a different set of $a_{n}$ and $b_{n}$ coefficients. Hence, it is an extremely fast operation, as fast as the tracking of one ray through the multipole.

This concludes our discussion of the computation of a map around a particular trajectory. In the remaining sections, we illustrate how one obtains invariants from the map around a closed orbit.

\section{A NONLINEAR FLOOUET REPRESENTATION}

As mentioned earlier, it is quite important to obtain the exact behavior resulting from misalignments or chromatic effects. In the study of the contemplated SSC, 
the chromatic effects of high multipole errors must be computed to high orders in $\delta$ or the misalignments. However, most of the properties around the resulting closed orbit can be explained by a relatively low-order study of the optics around it. In Appendix B, we apply this technique to a linear problem. As the linear problem is exactly solvable, it is an ideal example of the perturbative approach introduced in this section. This example is presented for pedagogical purposes only. In the following two sections, we will derive some of the nonlinear canonical transformations implemented in the program MARYLIE; they are used in obtaining invariants and tune shifts. Notice references to equations in Appendix B, which allow us to establish connections between the nonlinear case and the equivalent linear computation in Appendix B. A more exact link between the two computations is obtained if $g_{3}$ in Eq. (30) is set to zero. The reader is advised to set $g_{3}$ to zero (no sextupolar terms in the map) in the first reading of Sections 3 and 4 .

Traditionally, the computation of invariants and other nonlinear quantities requires the use of canonical transformations on the Hamiltonian. The Hamiltonian is $s$-dependent and so must be the transformations (this is true whether Lie methods or generating functions are used). This greatly complicates the task, especially if a complicated machine with errors is under study. To simplify the work, we divide it in two parts. First, we compute a mapping as described in the previous sections. Then we use $s$-independent algebraic transformations on the map itself. Because of this modular approach, we have succeeded in computing cubic, quartic, and even quintic invariants for arbitrarily complicated configurations of the SSC.

To clarify what $s$-independent canonical transformations on a map are, let us recall the linear case. If a linear map is obtained at a given $s$ (i.e., a matrix), one can immediately extract the fractional parts of the tunes and the quadratic invariants. These invariants reduce to the usual Courant-Snyder invariants if midplane symmetry is present locally. At that location, getting the linear lattice functions does not require a knowledge of the Hamiltonian! Our method is an extension of this approach to nonlinear mappings. Unlike previous authors, we are not limited to simple idealized rings (see Ref. 6).

To start, let there be a map $M$ expressed around its fixed point f. For example, $\mathcal{M}$ may represent the off-momentum motion with magnet errors for one turn of the machine. This map has a Lie algebraic representation:

$$
\begin{gathered}
\mathcal{M}=\exp \left(: f_{2}:\right) \exp \left(: f_{3}:\right) \exp \left(: f_{4}:\right), \\
f_{m}\left(\xi_{o}\right)=\sum_{i+j+k+l=m} A_{i j k l} x_{0}^{i} p_{x 0 y 0}^{j} p_{y 0}^{l} .
\end{gathered}
$$

Here the Lie operators are expressed in terms of the initial restricted phase-space vector $\xi_{0}$. For simplicity, we will not look at the full phase-space $\zeta_{0}$.

Using some standard techniques, it is possible to find a canonical transformation which transforms $f_{2}$ into a very simple operator: ${ }^{13}$

$$
\exists \mathscr{A}_{2} \text { such that } \mathscr{A}_{2} f_{2}=-\pi v_{x}\left(x_{0}^{2}+p_{x 0}^{2}\right)-\pi v_{y}\left(y_{0}^{2}+p_{y 0}^{2}\right)
$$


This transformation, when applied to $\mathcal{M}$, leads to the result

$$
\begin{aligned}
\mathscr{M}_{F}=\mathscr{A}_{2} \mathcal{M} \mathscr{A}_{2}^{-1} & =\exp \left(: \mathscr{A}_{2} f_{2}:\right) \exp \left(: \mathscr{A}_{2} f_{3}:\right) \exp \left(: \mathscr{A}_{2} f_{4}:\right) \\
& =\exp \left(: g_{2}:\right) \exp \left(: \mathrm{g}_{3}:\right) \exp \left(: g_{4}:\right) .
\end{aligned}
$$

We will say that $\mathcal{M}_{F}$ is the map $\mathcal{M}$ in linear Floquet variables. At this stage, it is possible to introduce the usual action-angle variables:

$$
\begin{gathered}
x=\sqrt{2 I} \cos \phi, \\
p_{x}=-\sqrt{2 I} \sin \phi, \\
y=\sqrt{2 J} \cos \psi, \\
p_{y}=-\sqrt{2 J} \sin \psi .
\end{gathered}
$$

The transformation of Eqs. (31) does not depend on the linear lattice functions since they are exterminated once and for all by $\mathscr{A}_{2}$ [Eq. (29)].

All the polynomials $g_{m}$ are rewritten as follows [see Eq. (B-6)]:

$$
g_{m}=\sum_{\substack{a+b=m \\|c|+|d|=m, m-2, \ldots}} \Gamma_{a b c d}^{m} I^{a / 2} J^{b / 2} \exp [i(c \phi+d \psi)],
$$

or, conveniently,

$$
g_{m}=\sum \Gamma_{a b c d}^{m}|a b c d\rangle
$$

In particular, $g_{2}$ has a simple form [see Eqs. (B-1a) and (B-1b)]:

$$
g_{2}=-2 \pi v_{x} I-2 \pi v_{y} J=-2 \pi v_{x}|2000\rangle-2 \pi v_{y}|0200\rangle \text {. }
$$

Moreover, the vector $|a b c d\rangle$ is an eigenvector of $: g_{2}:$. The application of $: g_{2}:$ on this vector gives the relation

$$
\begin{aligned}
: g_{2}:|a b c d\rangle & =\exp \left[i\left(c \mu_{x}+d \mu_{y}\right)\right]|a b c d\rangle, \\
\mu_{i} & =2 \pi v_{i} .
\end{aligned}
$$

The map $\mathscr{A}_{2}$ originally simplified the linear part of $\mathcal{M}$, allowing us to compute $\mathcal{M}_{F}$ in this new basis. Can we continue our simplification and reduce the nonlinear terms to their simplest form? To explore this, we apply a canonical transformation $\mathscr{A}_{3}$ on $M_{F}$ :

$$
\begin{aligned}
\mathscr{A}_{3} & =\exp \left(: F_{3}:\right) \\
\mathscr{M}_{F_{2}} & =\mathscr{A}_{3} \mathcal{M}_{F} \mathscr{A}_{3}^{-1} \\
& =\exp \left(: g_{2}:\right) \exp \left(:-g_{2}:\right) \mathscr{A}_{3} \exp \left(: g_{2}:\right) \exp \left(: g_{3}:\right) \exp \left(: g_{4}:\right) \mathscr{A}_{3}^{-1} .
\end{aligned}
$$

With the help of standard Lie algebraic tools, we rewrite Eq. (35b) accurately to the order of $g_{4}$ :

$$
\begin{aligned}
\mathcal{M}_{F_{2}}= & \exp \left(: g_{2}:\right) \exp \left\{: \exp \left(:-g_{2}:\right) F_{3}-F_{3}+g_{3}:\right\} \\
& \cdot \exp \left\{: g_{4}+\frac{1}{2}\left[\exp \left(:-g_{2}:\right) F_{3}, g_{3}-F_{3}\right]+\frac{1}{2}\left[F_{3}, g_{3}\right]:\right\} .
\end{aligned}
$$

The map $\mathcal{M}_{F_{2}}$ can be made simpler by removing its second-order content. This 
is done by requiring that its third-order polynomial vanishes:

$$
\left[1-\exp \left(:-g_{2}:\right)\right] F_{3}=g_{3} \text {. }
$$

It is easy to see that Eq. (37) can be inverted away from integer and third-integer resonances. This inversion is easily carried out using the eigenbasis representation:

$$
\begin{aligned}
F_{3} & =\left[1-\exp \left(:-g_{2}:\right)\right]^{-1} g_{2}, \\
& =\left[1-\exp \left(:-g_{2}\right)\right]^{-1} \sum_{\substack{a+b=3 \\
|c|+|d|=3,1}} \Gamma_{a b c d}^{3}|a b c d\rangle \\
& =\sum_{\substack{a+b=3 \\
|c|+|d|=3,1}} \frac{\Gamma_{a b c d}^{3}|a b c d\rangle}{1-\exp \left[-i 2 \pi\left(c v_{x}+d v_{y}\right)\right]} .
\end{aligned}
$$

The map $\mathcal{M}_{F_{2}}$ is purged from all quadratic contributions [see Eq. (B-1a)]:

$$
\begin{aligned}
\mathcal{M}_{F_{2}} & =\exp \left(: g_{2}:\right) \exp \left(: h_{4}:\right), \\
h_{4}= & g_{4}+\frac{1}{2}\left[F_{3}, g_{3}\right] \\
= & g_{4}+\frac{1}{2} \sum \frac{\Gamma_{a b c d}^{3} \Gamma_{\alpha \beta \kappa \delta}^{3}}{1-\exp \left[-i 2 \pi\left(c v_{x}+d v_{y}\right)\right]}[|a b c d\rangle,|\alpha \beta \kappa \delta\rangle], \\
{[|a b c d\rangle,|\alpha \beta \kappa \delta\rangle]=} & \frac{i}{2}(c \alpha-\kappa a)|(a+\alpha-2)(b+\beta)(c+\kappa)(d+\delta)\rangle \\
& +\frac{i}{2}(d \beta-\delta b)|(a+\alpha)(b+\beta-2)(c+\kappa)(d+\delta)\rangle .
\end{aligned}
$$

All of $h_{4}$ can be lumped into one sum [see Eq. (B-6)]:

$$
h_{4}=\sum_{\substack{a+b=4 \\|c|+|d|=4,2,0}} H_{a b c d}^{4}|a b c d\rangle .
$$

As we did before, we will attempt to simplify $\mathcal{M}_{F_{2}}$ with a canonical transformation $\mathscr{A}_{4}$ [see Eq. (B-5)]:

$$
\begin{gathered}
\mathscr{A}_{4}=\exp \left(: F_{4}:\right), \\
\mathcal{M}_{F_{3}}=\mathscr{A}_{4} \mathcal{M}_{F_{2}} \mathscr{A}_{4}^{-1}=\exp \left(: g_{2}:\right) \exp \left[: h_{4}+\exp \left(:-g_{2}\right) F_{4}-F_{4}:\right] .
\end{gathered}
$$

Here we discover that it is not possible to remove $h_{4}$ completely [Eqs. (B-6) and (B-7)]. In Eq. (40), the terms such that $|c|+|d|$ equals zero are not removable by canonical transformation. We separate them from the rest of $h_{4}$ :

$$
\begin{aligned}
h_{4}= & \sum_{\substack{a+b=4 \\
|c|+|d|=4,2}} H_{a b c d}^{4}|a b c d\rangle+H_{4000}^{4}|4000\rangle \\
& +H_{0400}^{4}|0400\rangle+H_{2200}^{4}|2200\rangle .
\end{aligned}
$$


As before, we can compute $F_{4}$ :

$$
F_{4}=\sum_{\substack{a+b=4 \\|c|+|d|=4,2}} \frac{H_{a b c d}^{4}}{1-\exp \left[-i 2 \pi\left(c v_{x}+d v_{y}\right)\right]}|a b c d\rangle .
$$

The final map is given by

$$
\begin{gathered}
M_{F_{3}}=\exp \left(: g_{2}:\right) \exp \left(: T_{4}:\right), \\
T_{4}=H_{4000}^{4}|4000\rangle+H_{0400}^{4}|0400\rangle+H_{2200}^{4}|2200\rangle \\
=H_{4000}^{4} I^{2}+H_{0400}^{4} J^{2}+H_{2200}^{4} I J .
\end{gathered}
$$

One notices that $g_{2}$ and $T_{4}$ are all expressed in terms of the action variables $I$ and $J$. Therefore, $: g_{2}:$ and $: T_{4}$ : commute, and the exponents in $\mathcal{M}_{F_{3}}$ can be combined [Eq. (B-8)]:

$$
M_{F_{3}}=\exp \left(: g_{2}+T_{4}:\right)
$$

It is a simple exercise to show that $T_{4}$ produces tune shifts with amplitude. In fact, the tune shifts are just [see Eq. (B-9)]:

$$
\begin{aligned}
\left(\begin{array}{l}
\Delta v_{x} \\
\Delta v_{y}
\end{array}\right) & =\left(\begin{array}{ll}
A & B \\
B & C
\end{array}\right)\left(\begin{array}{l}
I \\
J
\end{array}\right) . \\
A=-\frac{H_{4000}^{4}}{\pi}, \quad B & =-\frac{H_{2200}^{4}}{2 \pi}, \quad C=-\frac{H_{0400}^{4}}{\pi} .
\end{aligned}
$$

Finally, it is clear that near certain resonances the canonical transformations represented by $F_{3}$ and $F_{4}$ will be ill-defined. This is closely related to the absence of true nonlinear invariants of the map $\mathcal{M}$.

The quantity $\left(g_{2}+T_{4}\right)$ of Eq. (45) is not new. It is proportional to the Hamiltonian of the system after third-order $s$-dependent canonical transformations have cleaned up the original Hamiltonian. In practice, it is very hard to extract this quantity from the realistic Hamiltonian of the machine (realistic $H=$ realistic lattice file of the tracking program). For readers interested in deep connection, the author has proved the formal equivalence ${ }^{14}$ between a map algorithm and the Lie methods of Michelotti. ${ }^{5}$ In the next section, we derive a cubic and quartic correction to the linear invariants. The map $\mathcal{M}$ is also rewritten in terms of a pseudo-Hamiltonian $\mathbf{H} .^{15}$

\section{THE NONLINEAR INVARIANTS AND THE PSEUDO-HAMILTONIAN}

In the previous section, we computed the canonical transformations leading to $\mathcal{M}_{F_{3}}$. One can express the original map $\mathcal{M}$ in terms of $\mathcal{M}_{F_{3}}$ :

$$
\begin{gathered}
M=\mathscr{A}^{-1} \mathcal{M}_{F_{3}} \mathscr{A}, \\
\mathscr{A}=\exp \left(: F_{4}:\right) \exp \left(: F_{3}:\right) \mathscr{A}_{2} .
\end{gathered}
$$


Let us write two functions with the help of $\mathscr{A}$ :

$$
\begin{aligned}
& I_{4}=\mathscr{A}^{-1} I=\mathscr{A}^{-1}\left(\frac{x_{0}^{2}+p_{x 0}^{2}}{2}\right), \\
& J_{4}=\mathscr{A}^{-1} J=\mathscr{A}^{-1}\left(\frac{y_{0}^{2}+p_{y 0}^{2}}{2}\right) .
\end{aligned}
$$

It is easy to check that $I_{4}$ and $J_{4}$ are quartic invariants of $\mathcal{M}$ :

$$
\begin{aligned}
\mathscr{M I}_{4} & =\mathscr{A}^{-1} \mathcal{M}_{F_{3}} \mathscr{A} \mathscr{A}^{-1} I \\
& =\mathscr{A}^{-1} \mathcal{M}_{F_{3}} I \\
& =\mathscr{A}^{-1} \exp \left(: g_{2}+T_{4}:\right) I \\
& =\mathscr{A}^{-1} I=I_{4} ;\left[g_{2}, I\right]=\left[T_{4}, I\right]=0 .
\end{aligned}
$$

Because $g_{2}$ and $T_{4}$ are polynomials in $I$ and $J$, their Poisson bracket with $I$ or $J$ is zero. As an example, we can compute the cubic invariant $I_{3}$ :

$$
\begin{aligned}
I_{3} & =\mathscr{A}_{2}^{-1} \exp \left(: F_{3}:\right) I \\
& =\mathscr{A}_{2}^{-1}\left(I+\left[F_{3}, I\right]\right)+\cdots \\
& =\mathscr{A}_{2}^{-1}\left(I+\left[F_{3},|2000\rangle\right]\right) \\
& =\mathscr{A}_{2}^{-1} I+\mathscr{A}_{2}^{-1} \sum_{\substack{a+b=3 \\
|c|+|d|=3,1}} \frac{-\Gamma_{a b c d}^{3} i c|a b c d\rangle}{1-\exp \left[-i 2 \pi\left(c v_{x}+d v_{y}\right)\right]} \\
& =\mathscr{A}_{2}^{-1} I+\mathscr{A}_{2}^{-1} \Delta I_{3} .
\end{aligned}
$$

The quantity $\mathscr{A}_{2}^{-1} I$ is the quadratic invariant. The term $\mathscr{A}_{2}^{-1} \Delta I_{3}$ is the cubic correction to $\mathscr{A}_{2}^{-1} I$.

Finally, we introduced the concept of a pseudo-Hamiltonian. The pseudoHamiltonian $\mathbf{H}$ is not the Hamiltonian of the system. However, if a pseudo-time $\sigma$ is introduced and the pseudo-Hamiltonian is integrated from $\sigma=0$ to $\sigma=1$, this will reproduce the map $\mathcal{M}$. In a linear system, as seen in Appendix B, the pseudo-Hamiltonian is just proportional to the Courant-Snyder invariant. Clearly, it is not the Hamiltonian of the system.

We can compute the pseudo-Hamiltonian $\mathbf{H}$ using $\mathscr{A}$ [see Eq. (B-10)].

$$
\begin{aligned}
\mathcal{M} & =\mathscr{A}^{-1} \exp \left(: g_{2}+T_{4}:\right) \mathscr{A} \\
& =\exp \left(\mathscr{A}^{-1}: g_{2}+T_{4}: \mathscr{A}\right) \\
& =\exp \left[: \mathscr{A}^{-1}\left(g_{2}+T_{4}\right):\right] .
\end{aligned}
$$

From Eq. (51), we can read off $\mathbf{H}$ :

$$
\begin{aligned}
\mathbf{H} & =-\mathscr{A}^{-1} g_{2}-\mathscr{A}^{-1} T_{4} \\
& =2 \pi v_{x} I_{4}+2 \pi v_{y} J_{4}-H_{4000}^{4} I_{4}^{2}-H_{0400}^{4} J_{4}^{2}-H_{2200}^{4} I_{4} J_{4} .
\end{aligned}
$$

All the manipulation described in Sections 3 and 4 are performed by the program MARYLIE. MARYLIE can compute invariants, pseudo-Hamiltonian, and 
generating function representations of any map provided by a kick code. ${ }^{15}$ It handles the full six-dimensional representation of the map $\mathcal{M}$.

\section{CONCLUSION}

We have shown how it is possible to extract the Lie algebraic polynomials corresponding to the operations of a kick code. In particular, we have worked out the tools necessary to compute the six-dimensional lattice functions of an exact kick code. These techniques apply to simple codes like TEAPOT or to more complicated symplectic integrators. Indeed, $\mathrm{Neri}^{16}$ has shown that the various symplectic integrators amount to a succession of kicks, rotations, and drifts.

Our techniques are also applicable to codes using the approximate formula $\mathbf{P}=(1+\delta) \mathbf{r}^{\prime}$.

It is the author's belief that the most important contribution of the Lie algebraic methods to accelerator design resides in the analytical powers of the Lie algebraic tools which permit the extraction of tune shifts and invariants from complicated maps; in other words, in the context of large circular machines, the Lie algebraic manipulations capability of MARYLIE are more important than its ability to generate the map of a particular magnet. We hope that the reader will see that it is possible to extract from ordinary tracking codes the Lie algebraic polynomials of a transfer map.

\section{ACKNOWLEDGMENT}

The author is grateful to Richard Talman, Lindsay Schachinger, Albin Wrulich, Liam Healy, and Filippo Neri for discussing certain points of this paper. The author also acknowledges the work done by Alex Dragt's group at the University of Maryland. Many of the tools developed by the author are being improved in elegance and power by Dragt's group. In particular, they are extending the resonance basis subroutines to fifth order $\left(f_{6}\right)$.

\section{REFERENCES}

1. A. J. Dragt et al., IEEE Trans. Nucl. Sci., NS-32, 2311 (1985).

2. K. L. Brown, D. C. Carey, Ch. Iselin, and F. Rothacker, TRANSPORT, a Computer Program for Designing Charged Particle Beam Transport Systems, SLAC 91 (1973 rev.), NAL 91, and CERN 80-04.

3. K. L. Brown and R. V. Servranckx, First- and Second-Order Charged Particle Optics, SLAC-PUB-3381 (1984).

4. We reserve the words "nonlinear lattice functions" for the coefficients of a nonlinear invariant. These coefficients are a natural nonlinear extension of the linear Twiss parameters or linear lattice functions. (See Section 3).

5. R. D. Ruth, T. Raubenheimer, and R. L. Warnock, IEEE Trans. Nucl. Sci. NS-32, 2206 (1985).

6. Leo Michelotti, Particle Accelerators 16, 233 (1985).

7. H. Wiedemann, The Program PATRICIA, PEP note 220, Stanford Linear Accelerator Center (1979). 
8. A. Wrulich, RACETRACK, A Computer Code for the Simulation of Non-linear Particle Motion in Accelerators, DESY 84-026 (1984).

9. A. J. Dragt, Particle Accelerators 12, 205 (1982).

10. L. Schachinger and R. Talman, Particle Accelerators 22, 35 (1987).

11. One can transform a nonlinear matrix of the TRANSPORT type into a Lie algebraic representation. See, for example, E. Forest, D. Douglas, and B. Leemann, Study of the Aberrations of a Periodic Arc Using the Lie Algebraic Techniques, SSC-72 (1986), equation (3.7); also to appear in the Proc. of the Second Intern. Conf. on Charged Particle Optics.

12. David Douglas first derived this result for $P_{y i}=0$. Liam Healy recently noticed the intimate connection between $\mathscr{R}$ and the Hamiltonian written in polar $(r, \theta, z)$ coordinates. This connection allows for a general derivation of $\mathscr{R}$ in an arbitrary medium. Here, in Appendix A, we restrict ourselves to the case of a drift medium which is solvable by means of simple geometry.

13. E. Forest, Normal Form Algorithm on Non-linear Symplectic Maps, SSC-29 (1985).

14. E. Forest, Equivalence of Michelotti's Normal Form and the Map Normal Form as Used by the MARYLIE Code, SSC-30 (1985).

15. This work has been implemented in a version of MARYLIE used at the SSC Central Design Group. Efforts are under way to include these tools in the standard version of the code MARYLIE. It is also planned to extend these techniques to a fifth-order MARYLIE.

16. Neri has recently shown that the symplectic integrators of Ruth are just kick codes with a fancy splitting of the long elements into thin lenses. He rederived the results of Ruth with the help of the Campbell-Baker-Hausdorff theorem and proceeded to compute even higher-order integrators (private communication). See also R. D. Ruth, IEEE Trans. Nucl. Sci. NS-30, 2669 (1983).

17. A. J. Dragt, in Physics of High Energy Accelerators, AIP Conf. Proc. 87, 151 (1981).

18. A. J. Dragt and E. Forest, J. Math. Phys. 24, 2734 (1983).

19. It is possible to write a Hamiltonian for the process described by Eqs. (A-6) and (A-7) (Liam Healy, private communication). See Ref. 12.

20. See Ref. 16, p. 2623.

21. This index refers to the indexing system used in the program MARYLIE.

\section{APPENDIX A}

\section{The Drift $\mathscr{D}$ and the Rotation $\mathscr{R}$}

First, we must derive the map of Eq. (24). According to a standard result of Hamiltonian theory, the Hamiltonian ${ }^{17}$ for a drift is given by $-P_{z}$ :

$$
H=-P_{z}=-\left(1-\frac{2}{\beta} P_{t i}+P_{t i}^{2}-P_{x i}^{2}-P_{y i}^{2}\right)^{1 / 2} \text {. }
$$

Calling $\zeta_{i}$ the phase-space variables measuring deviations around $Z_{i}$, we can produce a Hamiltonian $K$ for the motion of $\zeta_{i}$ :

$$
K=-\left[1-\frac{2}{\beta} p_{\tau i}+p_{t i}^{2}-p_{x i}^{2}-\left(p_{y i}+P_{y i}\right)^{2}\right]^{1 / 2}-\left(\frac{p_{x i}}{\beta}+p_{y i} P_{y i}\right) \frac{1}{\sqrt{1-P_{y i}^{2}}} .
$$

The Lie algebraic polynomials for $\mathscr{D}$ are readily available from $K$. In fact, we need only to expand $K$ in powers ${ }^{18}$ of $\zeta_{i}$ :

$$
\begin{gathered}
k=\sum_{m=2}^{\infty} K_{m}, \\
f_{m}=-O B K_{m}, \\
\mathscr{D}\left(O B, P_{y i}\right)=\exp \left(: f_{2}:\right) \exp \left(: f_{3}:\right) \ldots
\end{gathered}
$$

The coefficients of $K_{3}$ and $K_{4}$ are listed in Table A-I as well as the matrix representation $M_{i j}$ of $\exp \left(:-O B K_{2}:\right)$. 
TABLE A-I

The Lie algebraic coefficients and the linear matrix of $\mathscr{D}\left(O B, P_{y i}\right)=\exp (:-O B k:)$

\begin{tabular}{cccccccl}
\hline Marylie Index $^{21}$ & $x^{i}$ & $p_{x}^{i}$ & $y^{k}$ & $p_{y}^{l}$ & $\tau^{m}$ & $p_{\tau}^{n}$ & \multicolumn{1}{c}{ Coefficients of $-K_{3}$ and $-K_{4}$} \\
\hline 51 & 0 & 2 & 0 & 1 & 0 & 0 & $-\left(b / 2 a^{3}\right)$ \\
53 & 0 & 2 & 0 & 0 & 0 & 1 & $-1 /\left(2 \beta a^{3}\right)$ \\
74 & 0 & 0 & 0 & 3 & 0 & 0 & $-\left(b / a^{3}+b^{3} / a^{5}\right) / 2$ \\
76 & 0 & 0 & 0 & 2 & 0 & 1 & $-\left(1 / a^{3}+3 b^{2} / a^{5}\right) /(2 \beta)$ \\
79 & 0 & 0 & 0 & 1 & 0 & 2 & {$\left[b / a^{3}-3 b /\left(\beta^{2} a^{5}\right)\right] / 2$} \\
83 & 0 & 0 & 0 & 0 & 0 & 3 & $-\left(\gamma^{-2}+\beta^{2} b^{2}\right) / 2 \beta^{3} a^{5}$ \\
140 & 0 & 4 & 0 & 0 & 0 & 0 & $-1 /\left(8 a^{3}\right)$ \\
149 & 0 & 2 & 0 & 2 & 0 & 0 & $-\left(1 / a^{3}+3 b^{2} / a^{5}\right) / 4$ \\
151 & 0 & 2 & 0 & 1 & 0 & 1 & $-3 b /\left(2 \beta a^{5}\right)$ \\
154 & 0 & 2 & 0 & 0 & 0 & 2 & {$\left[1 / a^{3}-3 /\left(\beta^{2} a^{5}\right)\right] / 4$} \\
195 & 0 & 0 & 0 & 4 & 0 & 0 & $-\left[1 /\left(8 a^{3}\right)+3 b^{2} /\left(4 a^{5}\right)+5 b^{4} /\left(8 a^{7}\right)\right]$ \\
197 & 0 & 0 & 0 & 3 & 0 & 1 & $-\left(3 b / a^{5}+5 b^{3} / a^{7}\right) /(2 \beta)$ \\
200 & 0 & 0 & 0 & 2 & 0 & 2 & $\left(1+3 b^{2} / a^{2}\right) /\left(4 a^{3}\right)-15 b^{2} /\left(4 \beta^{2} a^{7}\right)-3 /\left(4 \beta^{2} a^{5}\right)$ \\
204 & 0 & 0 & 0 & 1 & 0 & 3 & $-5 b /\left(2 \beta^{3} a^{7}\right)+3 b /\left(2 \beta a^{5}\right)$ \\
209 & 0 & 0 & 0 & 0 & 0 & 4 & {$\left[\left(-5+\beta^{2}\right) / \gamma^{2}-6 \beta^{2} b^{2}-\beta^{4} b^{4}+2 \beta^{4} b^{2}\right] /\left(8 \beta^{4} a^{7}\right)$} \\
\hline
\end{tabular}

$M_{i j}=$ matrix representation of $\exp \left(:-O B K_{2}:\right) ; M_{i i}=1, M_{12}=O B / a, M_{34}=O B\left(1 / a+b^{2} / a^{3}\right), M_{36}=$ $O B b /\left(\beta a^{3}\right), M_{56}=O B\left(\gamma^{-2}+\beta^{2} b^{2}\right) /\left(\beta^{2} a^{3}\right), M_{54}=O B b /\left(\beta a^{3}\right)$.

$b=P_{y i}, a=\left(1-P_{y i}^{2}\right)^{1 / 2}, \beta=v / c, \gamma=\left(1-\beta^{2}\right)^{-1 / 2}, k=$ Hamiltonian of a drift.

Because we have the Hamiltonian $K$, we are in a position to derive the rotation $\mathscr{R}$. Consider the rotation from $\zeta_{\perp}$ to $\zeta_{i}$ in Fig. 2. Calling $\mathbf{q}$ the vector $\left(x_{\perp}\right.$, $\left.y_{\perp}, \tau_{\perp}\right)$, we drift $\mathbf{q}$ from the plane of $\zeta_{\perp}$ to $\mathscr{P}_{i}$ :

$$
\begin{aligned}
\mathbf{q}_{\perp i} & =\exp (-: d K:) q_{\perp}, \\
d & =\tan \psi_{0} x_{\perp i} .
\end{aligned}
$$

Equation (A-4b) parametrizes the plane $\mathscr{P}_{i}$. It permits us to solve for $x_{\perp i}$. Notice that $d$ is the distance along the $O B$ direction of Fig. 2 and that $p_{s \perp}$ in the following equations is the total momentum along the same direction:

$$
\begin{gathered}
x_{\perp i}=\frac{x_{\perp}}{1-\frac{p_{x \perp}}{p_{s \perp}} \tan \psi_{0}}, \\
p_{s \perp}=\left[1-\frac{2}{\beta} p_{\tau \perp}+p_{\tau \perp}^{2}-p_{x \perp}^{2}-\left(p_{y \perp}+P_{y i}\right)^{2}\right]^{1 / 2} .
\end{gathered}
$$

Finally, we can use (A4) and (A5) to compute the vector $\mathbf{q}_{i}$ :

$$
\begin{gathered}
x_{i}=\frac{x_{\perp i}}{\cos \psi_{0}} \\
y_{i}=\left[\frac{p_{y \perp}+P_{y i}}{p_{s \perp}}\right] d+y_{\perp} \\
\tau_{i}=\left[\frac{\left(\frac{1}{\beta}-p_{\tau i}\right)}{p_{s \perp}}\right] d+\tau_{\perp} .
\end{gathered}
$$


TABLE A-II

The Lie algebraic coefficients and the linear matrix of $\mathscr{R}\left(\psi, P_{y i}\right)$

\begin{tabular}{cccccccl}
\hline Marylie Index $^{21}$ & $x^{i}$ & $p_{x}^{j}$ & $y^{k}$ & $p_{y}^{l}$ & $\tau^{m}$ & $p_{\tau}^{n}$ & \multicolumn{1}{c}{ Coefficients of $f_{3}$ and $f_{4}$} \\
\hline 34 & 1 & 2 & 0 & 0 & 0 & 0 & $-\tan \psi /(2 a)$ \\
35 & 1 & 1 & 1 & 0 & 0 & 0 & $-b \tan ^{2} \psi / a^{2}$ \\
38 & 1 & 1 & 0 & 0 & 0 & 1 & $-\tan ^{2} \psi /\left(\beta a^{2}\right)$ \\
43 & 1 & 0 & 0 & 2 & 0 & 0 & $-\tan \psi /(2 a)-b^{2}\left(\tan ^{3} \psi+\tan \psi\right) /\left(2 a^{3}\right)$ \\
45 & 1 & 0 & 0 & 1 & 0 & 1 & $-b\left(\tan ^{3} \psi+\tan \psi\right) /\left(\beta a^{3}\right)$ \\
48 & 1 & 0 & 0 & 0 & 0 & 2 & $-\left[\tan ^{2} \psi\left(\gamma^{-2}+\beta^{2} b^{2}\right)+\tan ^{3} \psi\right] /\left(2 \beta^{2} a^{3}\right)$ \\
105 & 1 & 3 & 0 & 0 & 0 & 0 & $-\tan ^{2} \psi /\left(4 a^{2}\right)$ \\
107 & 1 & 2 & 0 & 1 & 0 & 0 & $-\left(3 \tan ^{3} \psi+2 \tan \psi\right) b /\left(4 a^{3}\right)$ \\
109 & 1 & 2 & 0 & 0 & 0 & 1 & $-\left(\tan ^{2} \psi+3 \tan ^{3} \psi / 2\right) /\left(2 \beta a^{3}\right)$ \\
114 & 1 & 1 & 0 & 2 & 0 & 0 & $-\tan ^{2} \psi /\left(4 a^{2}\right)-b^{2}\left(3 \tan ^{4} \psi+5 \tan ^{2} \psi\right) /\left(4 a^{4}\right)$ \\
116 & 1 & 1 & 0 & 1 & 0 & 1 & $-b\left(3 \tan ^{4} \psi+5 \tan ^{2} \psi\right) /\left(2 \beta a^{4}\right)$ \\
119 & 1 & 1 & 0 & 0 & 0 & 2 & {$\left[\tan ^{2} \psi / a^{2}-\left(3 \tan ^{4} \psi+5 \tan ^{2} \psi\right) /\left(\beta^{2} a^{4}\right)\right] / 4$} \\
130 & 1 & 0 & 0 & 3 & 0 & 0 & $b^{2} \beta A+b \beta B$ \\
132 & 1 & 0 & 0 & 2 & 0 & 1 & $3 b A+B$ \\
135 & 1 & 0 & 0 & 1 & 0 & 2 & $3 A / \beta-b \beta B$ \\
139 & 1 & 0 & 0 & 0 & 0 & 3 & $-\left(\gamma^{-2}+\beta^{2} b^{2}\right) \tan ^{2} \psi /\left(2 \beta^{3} a^{5}\right)$ \\
& & & & & & & $+\tan ^{3} \psi\left[1-3 /\left(\beta^{2} a^{2}\right)\right] /\left(4 \beta a^{3}\right)-\tan ^{5} \psi /\left(4 \beta^{3} a^{5}\right)$ \\
\hline
\end{tabular}

$F_{i j}=$ matrix representation of $\mathscr{F}_{2}, G_{i j}=$ matrix representation of $\mathscr{G}_{2} ; F_{i i}=1, F_{24}=-b \tan \psi / a$, $F_{26}=-\tan \psi /(\beta a), F_{31}=b \tan \psi / a, F_{51}=\tan \psi /(\beta a), G_{11}=1 / \cos \psi, G_{22}=\cos \psi, G_{i i}=1(i=3,6)$. $b=P_{y i}, \quad a=\left(1-P_{y i}^{2}\right)^{1 / 2}, \quad \mathscr{R}=\mathscr{F}_{2} \quad e^{: f_{3}:} e^{: f_{4}: \mathscr{G}_{2}}, \quad A=-b\left(\tan ^{5} \psi / 2+3 \tan ^{3} \psi / 2+\tan \psi\right) /\left(2 \beta a^{5}\right), \quad B=-$ $\left(\tan \psi+\tan ^{3} \psi / 2\right) /\left(2 \beta a^{3}\right)$.

The momentum $p_{x \perp}$ must be redefined. This involves a simple rotation:

$$
\begin{aligned}
& p_{x i}=\cos \psi_{0} p_{x \perp}+\sin \psi_{0}\left[p_{s \perp}-\left(1-P_{y i}^{2}\right)^{1 / 2}\right], \\
& p_{y i}=p_{y \perp}, \\
& p_{\tau i}=p_{\tau \perp} .
\end{aligned}
$$

From Eq. (22), it is possible to find the Lie algebraic polynomial ${ }^{19}$ for $\mathscr{R}\left(\psi, P_{y i}\right)$. They are listed in Table A-II.

\section{APPENDIX B}

\section{A Linear Example of the Nonlinear Perturbation Theory}

Consider a map $M$ defined as follows:

$$
\begin{aligned}
\mathcal{M} & =\exp (:-\mu J:) \exp \left(: a x^{2}:\right), \\
J & =\frac{x^{2}+p^{2}}{2} .
\end{aligned}
$$

The parameter $a$ is assumed to be small. Nevertheless, it is possible to write $\mu$ with a single Lie operator. This is most easily seen by looking at the matrix 
representation of $\mathcal{M}$ :

$$
\begin{aligned}
\left(\begin{array}{l}
M x \\
M p
\end{array}\right)=M\left(\begin{array}{l}
x \\
p
\end{array}\right) & =\left(\begin{array}{cc}
1 & 0 \\
2 a & 1
\end{array}\right)\left(\begin{array}{cc}
\cos \mu & \sin \mu \\
-\sin \mu & \cos \mu
\end{array}\right)\left(\begin{array}{l}
x \\
p
\end{array}\right) \\
& =\left(\begin{array}{cc}
\cos \mu & \sin \mu \\
-\sin \mu+2 a \cos \mu & \cos \mu+2 a \sin \mu
\end{array}\right)\left(\begin{array}{l}
x \\
p
\end{array}\right) .
\end{aligned}
$$

It is well-known from the Courant-Snyder theory that the quadratic invariant of $M$ is just

$$
\begin{gathered}
I_{M}=\frac{1}{2}\left(\gamma x^{2}+2 \alpha x p+\beta p^{2}\right), \\
\alpha=-\frac{a \sin \mu}{\left[1-(\cos \mu+a \sin \mu)^{2}\right]^{1 / 2}}, \quad \beta=-\frac{\alpha}{a}, \quad \gamma=\frac{\alpha(-\sin \mu+2 a \cos \mu)}{a \sin \mu} .
\end{gathered}
$$

In terms of $I_{M}$, the map $M$ has the form ${ }^{20}$

$$
\begin{aligned}
\mu & =\exp \left(:-\tilde{\mu} I_{M}:\right), \\
\cos \tilde{\mu} & =\cos \mu+a \sin \mu .
\end{aligned}
$$

We can regain the results of Eqs. (B-3) and (B-4) by the perturbative techniques of Sections 3 and 4. Consider a transformation ordered in power of $a$ :

$$
\mathscr{A}=\cdots \mathscr{A}_{2} \mathscr{A}_{1}=\cdots \exp \left(: a^{2} F_{2}:\right) \exp \left(: a F_{1}:\right)
$$

First, we expand $a x^{2}$ in terms of the Floquet representation:

$$
\begin{aligned}
a x^{2} & =a 2 J \cos ^{2} \phi=\frac{a}{2} J\left(e^{i 2 \phi}+e^{-i 2 \phi}+2\right) \\
& =\frac{a}{2}(|22\rangle+|2-2\rangle+2|20\rangle) .
\end{aligned}
$$

The vector $|20\rangle$ in Eq. (B-6) is a tune shift term. The canonical transformation cannot remove $|20\rangle$ :

$$
\begin{aligned}
F_{1} & =\frac{1}{2} \frac{1}{1-\exp (:-\mu J:)}(|22\rangle+|2-2\rangle) \\
& =\frac{1}{2}\left[\frac{|22\rangle}{1-\exp (-i 2 \mu)}+\frac{|2-2\rangle}{1-\exp (i 2 \mu)}\right] .
\end{aligned}
$$

To first order in $a$, we have the equality:

$$
\begin{aligned}
\mathscr{A}_{1} \mathcal{M} \mathscr{A}_{1}^{-1} & =\exp (:-\mu|20\rangle:) \exp (: a|20\rangle:) \\
& =\exp [:(-\mu+a) J:] .
\end{aligned}
$$

From Eq. (B-8), we deduce the first-order shift of $\mu$. The result agrees with Eq. (B-4b):

$$
\tilde{\mu}=\mu-a+O\left(a^{2}\right) \cdots .
$$


Finally, we compute the pseudo-Hamiltonian of $\mathcal{M}$ using $F_{1}$ :

$$
\begin{aligned}
H & =\mathscr{A}_{1}^{-1}(\mu-a) J=(\mu-a)\left(J-a\left[F_{1}, J\right]\right) \\
& =(\mu-a)\left\{J-\frac{a}{2}\left[\frac{-2 i|22\rangle}{1-\exp (-i 2 \mu)}+\frac{2 i|2-2\rangle}{1-\exp (i 2 \mu)}\right]\right\} \\
& =(\mu-a)\left\{J+a J \frac{[\sin (2 \phi)-\sin (2 \phi+2 \mu)]}{1-\cos (2 \mu)}\right\} \\
& =\frac{(\mu-a)}{2}\left\{\left[1-\frac{a \sin (2 \mu)}{1-\cos (2 \mu)}\right] x^{2}+\left[1+\frac{a \sin (2 \mu)}{1-\cos (2 \mu)}\right] p^{2}-2 a x p\right\} .
\end{aligned}
$$

From Eq. (B-10), we deduce the value of the Twiss parameters:

$$
\begin{gathered}
\alpha=-a+O\left(a^{2}\right) \cdots, \\
\beta=1+\frac{a \cos u}{\sin \mu}+O\left(a^{2}\right) \cdots, \\
\gamma=1-\frac{a \cos \mu}{\sin \mu}+O\left(a^{2}\right) \cdots,
\end{gathered}
$$

These values agree to first order with Eq. (B-3). This concludes the treatment of this example. 